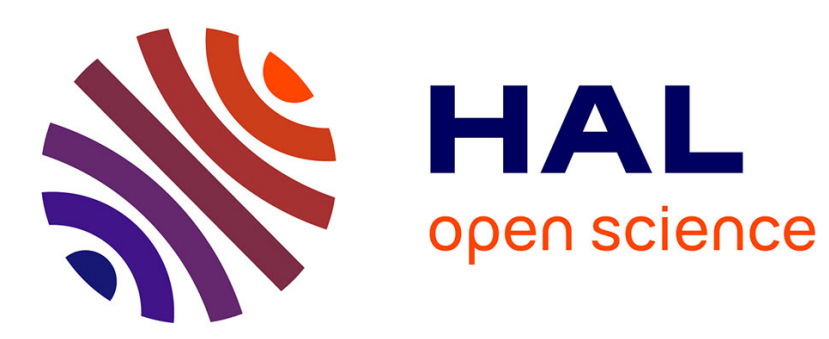

\title{
LA FONCTION PUBLIQUE FRANÇAISE AUX PRISES AVEC UNE DOUBLE EUROPÉANISATION
}

\author{
Jean-Michel Eymeri-Douzans
}

\section{To cite this version:}

Jean-Michel Eymeri-Douzans. LA FONCTION PUBLIQUE FRANÇAISE AUX PRISES AVEC UNE DOUBLE EUROPÉANISATION. Pouvoirs - Revue française d'études constitutionnelles et politiques, 2006, Serviteurs de l'Etat, 117 (2), pp.121. 10.3917/pouv.117.0121 . halshs-02914710

\section{HAL Id: halshs-02914710 \\ https://shs.hal.science/halshs-02914710}

Submitted on 13 Aug 2020

HAL is a multi-disciplinary open access archive for the deposit and dissemination of scientific research documents, whether they are published or not. The documents may come from teaching and research institutions in France or abroad, or from public or private research centers.
L'archive ouverte pluridisciplinaire HAL, est destinée au dépôt et à la diffusion de documents scientifiques de niveau recherche, publiés ou non, émanant des établissements d'enseignement et de recherche français ou étrangers, des laboratoires publics ou privés. 


\title{
LA FONCTION PUBLIQUE FRANÇAISE AUX PRISES AVEC UNE DOUBLE EUROPÉANISATION
}

\author{
Jean-Michel Eymeri-Douzans
}

Le Seuil | « Pouvoirs »

2006/2 n 117 | pages 121 à 135

ISSN 0152-0768

ISBN 9782020846271

Article disponible en ligne à l'adresse :

https://www.cairn.info/revue-pouvoirs-2006-2-page-121.htm

Distribution électronique Cairn.info pour Le Seuil.

(c) Le Seuil. Tous droits réservés pour tous pays.

La reproduction ou représentation de cet article, notamment par photocopie, n'est autorisée que dans les limites des conditions générales d'utilisation du site ou, le cas échéant, des conditions générales de la licence souscrite par votre établissement. Toute autre reproduction ou représentation, en tout ou partie, sous quelque forme et de quelque manière que ce soit, est interdite sauf accord préalable et écrit de l'éditeur, en dehors des cas prévus par la législation en vigueur en France. Il est précisé que son stockage dans une base de données est également interdit. 


\section{JEAN-MICHEL EYMERI}

\section{LA FONCTION PUBLIQUE FRANCCAISE AUX PRISES AVEC UNE DOUBLE EUROPÉANISATION}

"Je puis vous assurer que quand vous dites aujourd'hui que l'administration publique n'est pas une compétence communautaire, vous faites rigoler tout le monde.»

Propos d'un chef de bureau du ministère de la Fonction publique, septembre 2005 .

\section{A}

U SERVICE DE L'ÉTAT SONT DES SERVITEURS. Ce constat est d'évidence en tous pays, mais à l'évidence tous les États n'ont pas doté leurs agents d'un régime juridique exorbitant du droit commun du travail tel qu'en France, où les fonctionnaires - mot d'ailleurs intraduisible en maintes langues - sont soumis à un statut général qui définit les contours de la «Fonction publique». L'administration comparée ${ }^{1}$ montre d'ailleurs combien sont différenciées les formes d'organisation, les traditions et les usages de ces bureaucraties d'agents publics, au point que l'on peut repérer, avec Tocqueville, de véritables «constitutions administratives » propres à chaque État-nation, pour bonne part coutumières, et qui survivent souvent aux régimes politiques.

C'est pourquoi les administrations nationales ne sont pas en position semblable au regard des dynamiques transnationales qui les tou-

1. Dans cette veine, voir Françoise Dreyfus, L'Invention de la bureaucratie, La Découverte, 2000; Françoise Dreyfus, Jean-Michel Eymeri (dir.), Science politique de l'administration. Une approche comparative, Economica, 2006; Vincent Wright, Edward Page (éd.), Bureaucratic Elites in Western European States, Oxford, Oxford University Press, 1999. 
chent. Qu'en est-il sous ce rapport de la fonction publique française et de l'européanisation ? Pour apporter quelques linéaments de réponse, l'on soulignera d'abord que l'« européanisation » est un processus complexe et composite, dans lequel il est possible de distinguer une dynamique d'européanisation juridique et une dynamique d'européanisation sociologique, dont les effets seront envisagés tour à tour.

\section{UNE DOUBLE EUROPÉANISATION}

La notion très en vogue d' "européanisation » renvoie à des évolutions plus différenciées qu'il y paraît: en matière de fonction publique, elle recouvre une dualité de phénomènes qui doivent donc être distingués même s'ils convergent dans leurs effets ${ }^{2}$.

122 Tout d'abord, le droit de notre fonction publique est touché par des dispositions de droit communautaire dérivé qui ont remis en cause certains principes d'organisation et certaines règles de fonctionnement, contraignant les autorités françaises à des adaptations délicates. Il s'agit là d'une européanisation du et par le droit, dont la logique ne diffère guère de ce que l'on observe dans les autres branches du droit, et qui emporte bien sûr des conséquences très concrètes pour les acteurs.

En parallèle à cette européanisation juridique, il en existe une autre, qui tient à ce que s'est constitué un espace européen de politiques publiques. Il s'agit d'un ensemble polymorphe d'instances institutionnalisées (groupes de travail du Conseil, comités de comitologie, etc.) ou informelles (workshops divers, réseaux d'expertise, etc.), qui sont autant de forums de discussion et d'arènes de négociation générés par la «méthode communautaire». Ces espaces d'échanges relatifs aux politiques communautaires et aux éléments de convergence européenne des politiques nationales réunissent des acteurs aux légitimés hétérogènes : gouvernants politiques et fonctionnaires gouvernants des différents territoires et secteurs d'action publique, confédérations professionnelles et syndicales, médiateurs d'intérêts organisés et groupes mobilisés, experts et consultants en tout genre. Dans cet espace où l'on «s'entreglose» (pour le dire avec Montaigne), les interlocuteurs ne font pas toujours et jamais seulement de la production juridique. Sur le mode souple de la coopération intergouvernementale, les partenaires

2. Pour des réflexions sur ce lien, Jean-Michel de Forges, «Exigences communautaires et exigences managériales se rejoignent-elles ? ", $A J D A, \mathrm{n}^{\circ}$ 36, 27 octobre 2003, p. 1917-1923. 
présents échangent des expériences, exposent leurs best practices et leurs success stories, définissent des clusters et expérimentent des pilots, se soumettent à des exercices de benchmarking et de peer review, et convergent parfois sur des guidelines for action - l'anglais est utilisé non par pédanterie mais pour garder sa saveur au sabir communautaire. Ce faisant, les partenaires élaborent des problématiques convergentes, structurent des schèmes perceptifs, cognitifs et évaluatifs très proches, se dotent d'un sens commun partagé ${ }^{3}$. Ils convergent aussi sur des répertoires d'action concrets, des recettes organisationnelles, des registres d'instruments et d'outils privilégiés qu'ils se repassent (notre chèque emploi-service a ainsi été copié dans plusieurs États de l'UE), cette dimension pratico-instrumentale étant d'une grande importance ${ }^{4}$. L'on assiste ainsi à une européanisation conjointe des systèmes de croyances et de l'économie des pratiques des décideurs politico-administratifs, qui concourt à une sorte d' "esprit européen des lois », qui n'est pas le fruit d'obligations juridiques mais d'une socialisation mutuelle, à la fois dialogique et mimétique.

Pour ce qui est de la fonction publique, en tant que telle hors du champ de compétences de la Communauté défini par les traités, s'est mis en place depuis la décennie 1980 un réseau des ministères en charge de la fonction publique des États membres, animé par l'Institut européen d'administration publique (IEAP) implanté à Maastricht, aux PaysBas. Ce réseau interinstitutionnel est le cadre de travaux d'études et d'échanges dont l'observation ${ }^{5}$ indique qu'il fonctionne comme un des lieux de diffusion transeuropéenne du New Public Management (NPM). Ce constat vaut en particulier pour la France. Le NPM, élaboré dans les think tanks néo-libéraux états-uniens et britanniques des années 1980, a connu la diffusion internationale d'un "paradigme global " ${ }^{6}$, directe dans certains cas (entre pays anglo-saxons), mais le plus souvent médiatisée par les travaux de l'OCDE. Les responsables de notre administration étant rétifs envers l'OCDE et n'ayant guère recouru à des cabinets de consultants anglo-saxons, il semble que leur acculturation au néomanagérialisme soit surtout passée par les échanges au sein de l'espace

3. Yves Surel, «L'intégration européenne vue par l'approche cognitive et normative des politiques publiques ", RFSP, 50 (2), avril 2000, p. 235-254.

4. Pierre Lascoumes, Patrick Le Galès (dir.), Gouverner par les instruments, Presses de Sciences po, 2004.

5. L'auteur a été détaché de 1999 à 2001, comme représentant officiel français à l'IEAP.

6. Christopher Hood, "Contemporary Public Management : a new Global Paradigm ", Public Policy and Administration, 1995, p. 104-117. 
communautaire européen. Le même constat vaut pour cet autre pays de haute tradition administrative qu'est l'Allemagne, tard venu aux réformes administratives d'inspiration néo-managérialiste.

Qu'il s'agisse de l'une ou de l'autre de ces européanisations, il est patent que la fonction publique française se trouve atteinte, et doit bon gré mal gré s'adapter; pour autant, il ne semble pas qu'elle soit menacée dans sa survie ${ }^{7}$.

\section{L’EUROPÉANISATION PAR LE DROIT \\ ATTEINT CERTAINES SPÉCIFICITÉS \\ DE NOTRE FONCTION PUBLIQUE}

Le principe de libre circulation des travailleurs n'est, dit le traité de 124 Rome, «pas applicable aux emplois dans l'administration publique». Sur cette base, la CJCE a développé une conception duale de la fonction publique, contradictoire avec la tradition française. Notre construction statutaire, empreinte de l'égalité en droit issue de la Révolution, repose sur le principe que tous les agents publics participent peu ou prou à l'exercice des missions de la puissance publique, quel que soit leur niveau hiérarchique ou leur domaine d'emploi, et qu'ils doivent donc bénéficier d'un régime juridique commun. La France n'est pas isolée, puisque notre modèle a influencé l'Espagne, le Portugal, la Belgique, les Pays-Bas, l'Irlande ou la Grèce, tous pays où la qualité de fonctionnaire statutaire est accordée à tous les agents permanents des administrations.

À rebours, la CJCE a défini les «emplois dans l'administration publique» comme ceux qui «comportent une participation directe ou indirecte à l'exercice de la puissance publique et aux fonctions qui ont pour objet la sauvegarde des intérêts généraux de l'État et des autres collectivités publiques ». Dans cette conception fonctionnelle, c'est la nature des fonctions concrètement exercées qui est examinée et détermine le régime juridique applicable, ouvert ou fermé à la libre circulation selon que l'on touche à la puissance publique. La portée de cette distinction va bien au-delà de l'ouverture de tels emplois aux Européens non nationaux (réalisée par étapes : loi du 26 juillet 1991, décret du 6 janvier 2003, loi du 13 juillet 2005). Poser qu'au sein de ce que les États

7. Cette thèse nuancée est proche de celle de Marcel Pochard, «Les implications de la libre circulation: plus qu'une banalisation, la normalisation du droit de la fonction publique ", $A J D A, \mathrm{n}^{\circ} 36$, p. $1906-1910$. 
appellent «fonction publique» seul un noyau délimité travaille dans «l'administration publique », dont le statut des personnels peut être distinct, aboutit à mettre en question le statut exorbitant du droit du travail des nombreux agents employés hors de ce noyau sanctifié. Cela revient à poser la question: «Pourquoi les enseignants ou les personnels infirmiers sont-ils des fonctionnaires et non des agents contractuels ? » Question évidemment politique, induite par la comparaison européenne qui nous montre, au Royaume-Uni, dans les États scandinaves, en Italie désormais, que la majeure partie des agents du service public sont des contractuels de droit privé. Sous ce rapport, c'est sans doute avec le droit allemand (et autrichien et luxembourgeois), qui distingue le Beamte statutaire de l'employé public (Angestellte), que la construction duale et fonctionnelle du droit communautaire a le plus d'affinités.

Notre fonction publique évoluera-t-elle vers ce modèle où ne relèveraient du statut que les fonctionnaires d'autorité " participant à l'exercice de fonctions traditionnellement qualifiées de régaliennes ${ }^{8}$ ", tandis que les autres agents deviendraient contractuels ? Plus même, s'agiraitil de contrats de droit public, comme ceux des actuels agents non titulaires, ou d'une « privatisation de l'emploi public » telle que l'a connue depuis 1993 l'Italie, avec passage sous le régime du droit du travail de tous les agents (hormis les dirigenti des ministères, les préfets, les diplomates et les magistrats) ? À l'évidence, les conditions politiques de félicité d'une telle réforme sont différentes dans l'Italie de l'opération mani pulite et dans la France actuelle, où la capacité de mobilisation et le poids électoral des fonctionnaires sont considérables. Une réforme brutale semble improbable, tandis qu'une évolution graduelle vers la « dualisation» de l'emploi public paraît plausible.

Elle l'est d'autant plus que les avancées du droit communautaire obligent à améliorer les conditions d'emploi des contractuels. L'administration française a toujours eu recours à des agents non titulaires, «précaires perpétuels» liés avec l'employeur public par un contrat à durée déterminée, qui devait être renouvelé de façon expresse, et l'était le plus souvent, parfois pendant vingt ans. Or un accord-cadre européen - où les États employeurs, ô ironie, n'étaient pas représentés ! - a rappelé que le contrat à durée indéterminée est partout la norme, et une directive du 28 juin 1999 a donné pleine valeur à cet accord. En vertu de cette directive, les administrations doivent recruter leurs contractuels 
pour un CDD de trois années au maximum, renouvelable une seule fois, après quoi elles doivent les remercier ou transformer leur contrat en un CDI. Les autorités de notre fonction publique, soumises à un dialogue social permanent avec les syndicats de fonctionnaires (titulaires!), ont traîné les pieds dans la mise en conformité de notre droit interne, la loi de transposition n'étant enfin promulguée que le 26 juillet 2005. L'on pourra d'ici quelques années apprécier les effets de ces dispositions sur le développement (ou non) de la place des contractuels dans nos administrations.

De façon concomitante, l'européanisation du droit entre en contradiction avec l'organisation par corps de notre fonction publique, et sa focalisation sur le grade au détriment de l'emploi. Cela n'a rien de surprenant, puisque les corps administratifs, ces groupes de fonctionnaires 126 régis par le même statut particulier, et souvent soudés par une formation, un "esprit de corps", des intérêts communs, voire des stratégies collectives, sont une exception française qui n'a été copiée qu'en Espagne. Survivances de la société d'ordres et des corporations de métiers de l'Ancien Régime ${ }^{9}$, les corps ont une importance fondamentale dans notre sociologie administrative, et même dans la conduite de certaines politiques publiques. Leur nombre s'est démultiplié pour atteindre 1500 au milieu des années 1990, quand la Fonction publique décida d'engager une politique de fusions pour réduire ce chiffre extravagant. Quant à l'ancestrale distinction du grade et de l'emploi, elle est inconnue dans la plupart des administrations de l'UE. Dès lors, le droit communautaire, étranger à nos singularités, a imposé au législateur français de sortir d'un raisonnement par corps et par grades pour passer à un raisonnement par emplois. Ainsi, alors que la loi de 1991 et le décret de 2003 s'en tenaient au principe d'ouvrir des corps entiers aux ressortissants communautaires ou de les maintenir fermés en bloc si certains emplois auxquels ils donnent accès touchent à l'exercice de la puissance publique, les critiques réitérées de la Commission et de la CJCE ont conduit le législateur français à résipiscence. La loi du 26 juillet 2005 dispose que tous les corps sont en principe ouverts aux Européens, et qu'il faut apprécier au cas par cas si tel emploi comporte une dimension de puissance publique telle qu'il faille le réserver à nos nationaux. C'est là une petite révolution par rapport au modèle traditionnel et aux routines sédimentées : le droit de la fonction publique dit désormais que

9. Dominique Chagnollaud, Le Premier des ordres, Fayard, 1991. 
la gestion individualisée des carrières, au cas par cas, est possible et même parfois indispensable. Comme c'est aussi ce que répètent à satiété les conseils en management des ressources humaines, quoi d'étonnant à ce que Marcel Pochard, ancien directeur général de l'administration et de la fonction publique, dans le rapport public 2003 du Conseil d'État, ait mis à l'agenda le projet d'abolir ce millier de corps administratifs pour regrouper les fonctionnaires en une cinquantaine de vastes «cadres de fonctions» ou «cadres statutaires» au sein desquels la gestion des personnels serait déconcentrée aux responsables de terrain, c'est-à-dire individualisée ${ }^{10}$. La conscience des lourdeurs de l'organisation actuelle est bien sûr primordiale dans ce projet, mais la logique du droit communautaire y a aussi sa part.

À cela s'ajoute que le droit communautaire de l'équivalence des qualifications et des diplômes remet en cause certaines modalités du recrutement de nos fonctionnaires. Ce n'est pas, comme l'ont écrit certains, le principe du concours qui serait menacé. Certes la France est la patrie des concours, mais cette forme de recrutement ne nous est pas spécifique : hormis les quelques fonctions publiques dite «d'emploi » (Suède, Finlande, Danemark, et Royaume-Uni plus récemment), la plupart des pays de l'UE, et les institutions communautaires mêmes, recrutent leurs agents publics titulaires par des concours. Notre spécificité gît dans l'existence d'écoles administratives, qu'il s'agisse de grandes écoles prestigieuses (école des Mines, ENA...) ou d'institutions de moindre prestige (École nationale de la santé publique de Rennes, écoles de police, IRA, etc.). Ces écoles, inconnues ailleurs, ont la particularité d'être distinctes des universités, d'être peuplées de praticiens qui y forment leurs futurs jeunes collègues sur un mode mimétique, de ne délivrer aucun diplôme, d'avoir un concours d'entrée qui fonctionne comme un concours de recrutement des corps auxquels donne accès l'école, et un concours ou classement de sortie sur épreuves qui détermine les affectations d' «élèves » qui sont dès le départ des fonctionnaires stagiaires ${ }^{11}$.

C'est cette « exception française » que l'arrêt Burbaud de la CJCE du 9 septembre 2003 est venu frapper de plein fouet ${ }^{12}$. Tout d'abord, en vertu des règles d'équivalence générale des diplômes, la CJCE requalifie

10 Conseil d'État, Rapport public 2003, La Documentation française, 2003, p. 344 et s.

11. Sur ces écoles administratives dont l'archétype est l'ENA, Jean-Michel Eymeri, La Fabrique des énarques, Economica, 2001.

12. Pour les détails, voir Fabrice Melleray, «L'arrêt Burbaud implique-t-il une discrimination à rebours ? ", $A J D A, \mathrm{n}^{\circ} 36$, p. 1911-1916. 
en « diplôme» l'examen de sortie de notre École de santé publique. Puis, au titre de la libre circulation des travailleurs, la Cour affirme qu'un citoyen européen titulaire d'une formation ou diplôme équivalent dans un autre État membre ne saurait se voir imposer, pour intégrer le corps des directeurs d'hôpitaux, de passer par l'École de Rennes. C'est tout le système français des écoles administratives qui se trouve ébranlé, et qui n'a pas encore fini, trois ans après, d'absorber l'onde de choc. Car si les examens ou concours de sortie des IRA, de l'École des douanes, de l'ENA, etc., sont désormais des diplômes au sens matériel et communautaire, il faut donc leur conférer cette nature au sens formel et francofrançais. Dès lors se posent les difficultés de la «diplomation » : les écoles administratives, qui ne sont pas des établissements d'enseignement supérieur, peuvent-elles délivrer des diplômes universitaires sans un 128 corps d'enseignants permanents ? Faut-il hiérarchiser ces diplômes d'écoles en fonction de leur prestige et du niveau requis à leur entrée, les unes délivrant des licences quand les autres délivreraient des masters ? La chose n'est pas si simple puisque même la prestigieuse ENA n'exige en théorie qu'une licence à l'entrée, et encore pour les élèves externes. Plus avant, dans quelles disciplines seront délivrés ces diplômes ? Des disciplines existantes (droit, économie, science politique...) ? Mais les universités accepteront-elles que le diplôme de sortie de l'École des impôts soit une «maitrise en droit fiscal » ? S'orientera-t-on plutôt vers des diplômes propres, tel un "master en administration publique» décliné en spécialités selon les écoles considérées ? Ce dossier complexe présente une réelle dangerosité pour les décideurs politico-administratifs, d'autant que se profile la perspective que ces établissements délivrent des doctorats d'expertise...

Au-delà, la jurisprudence Burband touche à l'économie profonde des écoles administratives et à leur imbrication avec les corps. Car la CJCE commande que les ressortissants européens dans le cas de $\mathrm{M}^{\mathrm{me}}$ Burbaud, dispensés du passage par les écoles, soient inscrits sur la liste de classement final en vertu de laquelle les élèves sortants choisissent, par ordre de mérite, leurs affectations. Or le problème de savoir à quel rang classer les Européens ainsi «rajoutés» est indécidable. Dès lors, deux options ont été envisagées. La première option a été suggérée par l'avocate générale de la CJCE: il s'agirait de transformer nos écoles en instituts publics de formation spécialisée, peuplés d'étudiants auxquels serait délivré un diplôme final nécessaire pour se présenter à un concours de recrutement qui n'interviendrait qu'ensuite et serait ouvert sur un pied d'égalité aux titulaires de diplômes étrangers recon- 
nus équivalents. Cette réforme radicale a été écartée par le ministère de la Fonction publique. Une option plus plausible a été avancée par JeanMichel de Forges ${ }^{13}$ : instituer, en parallèle aux concours de sortie des écoles, des concours spéciaux d'accès direct aux mêmes corps réservés aux candidats déjà qualifiés, en nombre suffisant pour ne pas encourir le grief de pratiquer la «discrimination indirecte ", et ouverts tant aux ressortissants d'autres pays de l'UE qu'aux nationaux français, pour éviter la «discrimination à rebours". Toutefois, le problème d'avoir à réunir les lauréats des deux concours sur une liste de classement unique ne s'en trouve pas réglé ${ }^{14}$. Fort de ce constat, le ministère de la Fonction publique s'est arrêté à l'argumentaire que la jurisprudence Burbaud était inapplicable: des tractations ont lieu depuis des mois avec la Commission européenne (DG marché intérieur et DG emploi) aux fins qu'elle lance un projet de directive autorisant un État qui ouvre un concours spécial pour les ressortissants communautaires à qualification équivalente à les classer sur une liste distincte. Dans le cadre de "paquets » complexes de négociation entre la Commission et les autorités françaises, celles-ci pensent avoir (à l'heure où sont écrites ces lignes) des chances raisonnables de sauvegarder ainsi l'originalité du modèle français d'écoles administratives.

Quoi qu'il doive en être, il importe dans le même temps de mesurer combien les divers effets de cette européanisation juridique se trouvent redoublés dans leur efficace concret par une dynamique concomitante.

\section{L'EUROPÉANISATION SOCIOLOGIQUE CONTRIBUE À TRANSFORMER \\ NOTRE CULTURE ADMINISTRATIVE}

L'acculturation des responsables français au New Public Management n'a pas été immédiate: il leur a fallu bien plus de temps que leurs collègues des autres pays. Et cette appropriation, moins que d'une influence directe des modèles britannique, canadien ou néo-zélandais, souvent raillés par nos énarques pour leurs excès, a surtout résulté de la co-production par les partenaires de l'espace européen d'action publique d'une sorte de sens commun de la réforme administrative dont

13. Jean-Michel Lemoyne de Forges, L'Adaptation de la fonction publique française au droit communautaire : rapport au ministre de la Fonction publique, Dalloz, 2003.

14. Fabrice Melleray, art. cit., p. 1914. 
les préceptes et recettes néo-managérialistes, atténués dans leurs excès ${ }^{15}$ et saupoudrés d'un peu d'administration électronique (e-Government), semblent constituer le ressort principal.

La force du NPM lui vient sans doute d'être à la fois une grammaire mentale et comportementale. Il a bien sûr une dimension idéelle, voire idéologique: négation de toute distinction d'essence entre l'entreprise privée et les organisations publiques; redéfinition subséquente du citoyen assujetti et/ou usager en un client-consommateur; jugement négatif sur l'organisation présente des institutions publiques; affirmation que les solutions "qui marchent» dans le privé doivent être importées dans le public; et conviction qu'une efficacité accrue des administrations découlera de telles réformes principalement organisationnelles ${ }^{16}$. Toutefois, la force du néo-managérialisme est d'opéra130 tionnaliser ce corps de doctrine, de plus en plus souvent reçu pour vrai, dans et par un répertoire de recettes, de techniques et d'instruments (agencification, benchmarks, tableaux d'indicateurs, accréditation, contrôle de gestion, management par objectifs, guichets uniques, partenariats public-privé, évaluation ex ante, in itinere et ex post, etc.) érigés en «bonnes pratiques» qui objectivent le tournant néo-managérial des administrations publiques.

Dans ce contexte général, l'orientation prise depuis plusieurs années en France, à l'unisson de nos voisins, tend à rapprocher les conditions d'emploi des agents publics de celles des salariés du secteur concurrentiel. Cette évolution s'opère à petits pas et à bas bruit, sans annoncer à grand son de trompe ni une «privatisation de l'emploi public» à la manière de l'Italie de Berlusconi ni même une «normalisation» à la manière des autorités néerlandaises. L'on parle ici d'instaurer une « véritable gestion des ressources humaines », d'ajuster les effectifs recrutés aux besoins réels des services en se déprenant des ancestrales logiques corporatives, de bâtir une "gestion prévisionnelle des effectifs, des emplois et des compétences» (GPEEC), de déconcentrer les affectations et les mutations aux gestionnaires de terrain pour flexibiliser l'ensemble, de généraliser les répertoires de métiers et les fiches de poste permettant d'autant mieux que les affectations optimisent "l'adéquation profilposte » que les vacances d'emploi seront publicisées de manière enfin

15. Pour un exposé radical, David Osborne, Ted Gaebler, Reinventing Government, Reading, Addison-Wesley, 1992.

16. Christopher Pollitt, Managerialism and Public Services, Oxford, Blackwell, 1993. 
transparente dans des «bourses de l'emploi public» et que les «viviers administratifs » seront décloisonnés pour supprimer les «chasses gardées » corporatives. Plus avant, il s'agirait à terme de permettre aux chefs de services de motiver chaque agent public au moyen d'une panoplie d'outils managériaux : passation d'un contrat d'objectifs lors de la prise de poste, entretien annuel d'évaluation mesurant l'atteinte de ces objectifs, répercussions financières par l'introduction d'un complément de rémunération variable en fonction des résultats (performance-related pay). Certes, la réalité administrative française est loin de correspondre encore à ce schéma déjà mis en œuvre chez nos voisins plus septentrionaux, et l'histoire atteste que notre administration ne se transforme qu'avec lenteur. Mais telle est la direction dans laquelle travaillent les autorités de la Fonction publique, avec une intéressante constance audelà des alternances gauche-droite, sans l'afficher pour cause d'hostilité des principales organisations syndicales.

Aussi gradualiste soit-elle, cette évolution tend à rompre avec la structuration du monde administratif français héritée du passé. Pour l'exprimer avec quelque stylisation idéal-typique, notre fonction publique est un univers de règles et de rangs, de corporations et de privilèges, dont l'équilibre dynamique repose sur une collectivisation de la ressource principale: des emplois et les profits qui s'y attachent, qu'ils soient matériels (traitements aujourd'hui, pensions demain) ou symboliques («l'honneur social» qu'analysait Weber). Or, en comparaison d'autres pays, ce qui frappe dans l'administration française est la nature duale de cette ressource : elle est à la fois très hiérarchisée et très collectivisée... ce second trait rendant le premier acceptable. Notre administration est très hiérarchique : c'est le système des grandes écoles et des prestigieux concours qui confère à ses heureux élus une sorte de noblesse à titre viager ${ }^{17}$, c'est l'empilement des grands corps et moins grands corps issus de l'ENA et de l'X dont la domination est globale, mais aussi des «petits grands corps » dont la domination se limite à tel segment (les professeurs agrégés du secondaire par différence avec les certifiés, etc.), ces hiérarchies se déroulant jusqu'à la base. Notre fonction publique est encore largement une société d'ordres (au sens de l'Ancien Régime) organisée en corps dont chacun veille à "tenir son rang», c'est-à-dire à maintenir ses privilèges. Or ce ne sont pas des individus qui sont ainsi hiérarchisés et privilégiés, mais des groupes de statut, des

17. Voir Pierre Bourdieu, La Noblesse d'État, Minuit, 1989. 
collectifs, et sans doute est-ce cette dimension collective qui a permis que perdure depuis deux siècles au cœur de la République une telle société d'ordres. L'on retrouve en cela la dialectique tocquevillienne: passion de l'égalité et/mais goût des privilèges ${ }^{18}$. Le compromis historique, le contrat social pratique qui fonde notre fonction publique concilie ces deux tendances : le maintien d'une société d'ordres et de privilèges grâce à la collectivisation des ressources fondamentales. À la différence des traditions allemande et britannique, l'octroi généralisé dès le XIX ${ }^{\mathrm{e}}$ siècle aux agents de tous niveaux, du conseiller d'État jusqu'au facteur, du statut de fonctionnaire a collectivisé au profit de la masse des employés publics la ressource rare qu'est un emploi garanti à vie, indépendamment du travail fourni et des préférences politiques, en fait indépendamment de l'idiosyncrasie des individus. On l'a assorti de 132 traitements qui, sur longue période, ont été plus élevés que ceux des emplois comparables du secteur privé. S'y ajoutèrent de très favorables pensions de retraite, qui ont mis à l'abri des aléas de la vie ce vaste groupe social des fonctionnaires et de leurs familles, bien avant la Sécurité sociale, et souvent sur plusieurs générations vu la forte hérédité professionnelle dans l'administration, facilitant l'ascension sociale intergénérationnelle dans ce milieu. Ainsi, en conformité avec la mythologie politique de l'égalitarisme républicain, la base de la pyramide administrative a bénéficié d'un droit impersonnel de jouissance collectivisée d'une ressource précieuse. Aux divers étages supérieurs, ce système s'est répliqué sous la forme de privilèges (de rémunération, de carrière, de conditions de travail, de prestige, etc.) accordés non pas intuitu personae - ce serait le règne, tant dénoncé, de la «faveur » arbitraire -, mais reconnus et garantis à des corps, à des collectifs statutaires, d'autant plus légitimes que l'accès s'y fait conformément à la méritocratie républicaine par la réussite à des concours, dont certains sont même « internes » et permettent aux subordonnés les plus méritants de s'élever. Ainsi, que ce soient le pourcentage que les trésoriers-payeurs généraux empochent sur les impôts prélevés ou les indemnités des hauts administrateurs qui, à Bercy, doublent leur traitement grâce à des primes dont on peine à admettre qu'elles ne récompensent aucune performance particulière, ces avantages profitent à tous les membres du groupe. En France, les réussites administratives sont individuelles, mais les privilèges sont collectifs. Les carrières se situent d'ailleurs à l'intersection de 
ces deux dimensions : il n'est que de voir combien les plus grands corps gèrent de façon collective la carrière de chacun de leurs membres dans la perspective de l'intérêt du corps dans son ensemble (occupation de postes tenus pour des «chasses gardées ", etc.). Même le "pantouflage », cette autre spécificité française consistant pour un haut fonctionnaire à partir en cours de carrière vers une grande entreprise, a fait l'objet au $\mathrm{XX}^{\mathrm{e}}$ siècle (c'est moins vrai désormais) d'une gestion collective, le directeur du Trésor plaçant dans les grandes banques ses sous-directeurs expérimentés tandis que le président du conseil général des Ponts et Chaussées orchestrait la noria d'ingénieurs X-Ponts entre les DDE et les sociétés d'autoroute ou les entreprises du BTP. Cette gestion collective de leurs ressources par les grands corps d'État fait d'ailleurs écho au «collectivisme pratique» d'une grande bourgeoisie qui les peuple encore pour partie ${ }^{19}$. C'est ainsi que la fonction publique française, société d'ordres organisant un système de privilèges corporatifs sur un socle impersonnel de généreuse collectivisation de la ressource de base, a longtemps assuré la coexistence voire la collusion d'une masse de fonctionnaires issus des classes populaires et moyennes de sensibilité radicale à socialiste, très soucieuses d'égalité, et d'une haute fonction publique encore souvent issue de la haute bourgeoisie ou, a minima, de vieilles familles notabiliaires de province, très soucieuses du maintien des distinctions sociales.

L'on comprend que les orientations actuelles vers une nouvelle gestion des ressources humaines dans notre administration soient porteuses d'une déstabilisation en profondeur de ce compromis de base. Car la logique néo-managérialiste, forme du libéralisme contemporain, consiste à rechercher la maximisation de la performance des appareils institutionnels par l'individualisation: individualisation du recrutement, individualisation des conditions d'emploi, individualisation des affectations, individualisation des parcours de carrière, et surtout individualisation des rétributions et des sanctions (notamment pécuniaires). Là où notre tradition privilégiait la garantie contre les aléas et la stabilité des positions par l'indifférenciation et l'impersonnalité d'une gestion collective - prévisibilité que Weber voyait comme consubstantielle à la bureaucratie -, les innovations néo-managérialistes n'ont de cesse que d'introduire de l'aléa et de l'impermanence

19. Michel Pinçon, Monique Pinçon-Charlot, Sociologie de la bourgeoisie, La Découverte, 2000, p. 103. 
afin de mettre les individus en tension - tension supposée motrice et génératrice de performance. La logique sous-jacente est proche de celle à l'œuvre plus généralement, qui tend à faire peser sur les individus peuplant les organisations la charge principale de leur adaptation au fonctionnement des sociétés de marché contemporaines ${ }^{20}$. Cette orientation néo-managérialiste, si elle se maintient dans la durée, aboutira sans doute à transformer les grands équilibres et la culture au travail de notre fonction publique, à la base comme en son sommet. Les «petits » fonctionnaires devront s'accoutumer à une croissante intranquillité marquée par les impératifs catégoriques de la flexibilité, du rendement et de la qualité de service ${ }^{21}$. Les hauts fonctionnaires devront abdiquer maints privilèges, compter moins sur l'esprit de corps que sur l'esprit d'initiative, et surtout accepter de travailler et de faire carrière dans un

134 milieu désormais exposé au risque de la concurrence. Ils devront y refaire au quotidien et en pratique la preuve de leur excellence, en situation d'expertise désormais contestée tout à la fois par d'autres hauts fonctionnaires du fait du «décloisonnement des viviers » et des carrières, par des experts contractuels de haut niveau de plus en plus nombreux et «pointus », par des fonctionnaires territoriaux de mieux en mieux formés et de plus en plus influents, par des consultants privés de plus en plus sollicités car ils ciblent de mieux en mieux leur offre sur le secteur public, et encore par les contre-experts que mobilisent avec une efficacité grandissante les organisations dites « de la société civile» impliquées dans la production et la mise en ouvre interactionnelles des politiques publiques.

En fin de compte, tout semble se passer comme si les dynamiques conjointes d'européanisation juridique et sociologique, entrant en résonance avec d'autres facteurs tel le formidable renouvellement démographique de nos administrations dans les années qui viennent, et la relève des générations qu'il implique, alimentaient un processus de transition historique dont le rythme et l'ampleur nous sont bien sûr inconnus - les historiens l'apprécieront ex post -, mais qui semble faire évoluer la fonction publique française du règne de la prévisibilité centrée sur les règles et les rangs, sur le respect du droit, des droits acquis et des privilèges vers le règne des valeurs plus labiles de performance,

20. Luc Boltanski, Ève Chiapello, Le Nouvel Esprit du capitalisme, Gallimard, 1999.

21. Lire, sur ce sujet, Vincent Dubois, La Vie au guichet, Economica, 1999; Jean-Marc Weller, L'État au guichet, Desclée de Brouwer, 1999. 
d'adaptabilité et de mobilité, marqué par le recours grandissant à l'individualisation sur fond de "culture du chiffre», de batteries d'indicateurs, d'obligations de résultat et d'impératif d'évaluation. Il reste que le sociologue aura à observer et à expliquer comment et dans quelle mesure notre fonction publique, qui demeure un univers gorgé de juridicité, de traditions et de routines, recalcifiera sans doute pour partie cette labilité, illustrant la propension constante des univers bureaucratiques, bien attestée par les travaux de Weber, de Merton et de Crozier, à fabriquer du vieux avec du neuf.

Notre fonction publique est soumise à un double mouvement d'européanisation, juridique et sociologique, qui, sans mettre en péril ses grandes règles (existence du statut, règne du concours...), remet en cause plusieurs éléments de son "compromis historique» fondamental et alimente ainsi un processus de transition historique vers un modèle de fonction publique pour partie renouvelé, dont les contours sont encore imprécis même si l'orientation générale est perceptible. 